\title{
EQUIVARIANT BORDISM AND SMITH THEORY. II
}

\author{
R. E. STONG( ${ }^{(1)}$
}

\begin{abstract}
This paper analyzes the homomorphism from equivariant bordism to Smith homology for spaces with an action of a finite group $G$.
\end{abstract}

1. Introduction. Let $G$ be a finite group, and let $(X, A, \psi)$ be a pair with $G$ action. One then has defined the $G$-equivariant bordism group $\mathfrak{N}_{*}^{G}(X, A, \psi)$ and the Smith homology group $H_{*}^{G}\left(X, A, \psi ; Z_{2}\right)$. These define equivariant homology theories on the category of $G$ pairs and $G$-equivariant maps, and the object of this paper is to explore the relationship between these theories.

Briefly, being given an equivariant bordism element $f:(M, \partial M, \varphi) \rightarrow(X, A, \psi)$, the image of the fundamental Smith theory class of $(M, \partial M, \varphi)$ gives a natural transformation

$$
\tilde{\mu}: \mathfrak{N}_{*}^{G}(X, A, \psi) \otimes_{\mathfrak{N} q} Z_{2} \rightarrow H_{*}^{G}\left(X, A, \psi ; Z_{2}\right) .
$$

It was shown in [9] that $\tilde{\mu}$ is an isomorphism if $G=Z_{2}$.

The main results of this paper are

THEOREM 1. $\tilde{\mu}$ is always epic and

THeORem 2. $\tilde{\mu}$ is an isomorphism for all $G$ pairs $(X, A, \psi)$ if and only if $G$ is 2-nilpotent and has Sylow 2 subgroup a $Z_{2}$ vector space.

( $G$ is called 2-nilpotent if the elements of odd order in $G$ form a subgroup.)

2. The representation theorem. Let $G$ be a finite group, $X$ a simplicial complex, $\psi: G \times X \rightarrow X$ a simplicial $G$ action and $A \subset X$ a subcomplex invariant under $G$. It will be assumed that $X$ is "finely" triangulated so that the fixed set of any subgroup $H$ is a subcomplex and the projection $\pi: X \rightarrow X / H$ is simplicial (E. E. Floyd [4] shows that this may be accomplished by taking the second barycentric subdivision).

Let $C(X) \otimes Z_{2}$ denote the chains of $X$ with $Z_{2}$ coefficients and let $g_{\#}: C(X) \otimes Z_{2}$ $\rightarrow C(X) \otimes Z_{2}$ be the chain map induced by $\psi(g):, X \rightarrow X: x \rightarrow \psi(g, x)$. One then lets $C^{0}(X) \subset C(X) \otimes Z_{2}$ denote the subgroup consisting of chains $\sigma$ so that

Received by the editors March 1, 1971.

AMS 1969 subject classifications. Primary 5710; Secondary 5747.

Key words and phrases. Equivariant bordism, Smith theory.

${ }^{1}$ ) I am indebted to the Alfred P. Sloan Foundation for financial support during this work. Copyright (C) 1972, American Mathematical Society 
$g_{\#} \sigma=\sigma$ for all $g \in G$. Since $g_{\#}$ commutes with the boundary, one has an induced boundary $\partial: C^{0}(X) \rightarrow C^{0}(X)$, and if $C^{0}(X, A)=C^{0}(X) / C^{0}(A)$ one has induced a homomorphism $\partial$ making this a chain complex. The Smith homology groups of $(X, A, \psi), H_{*}^{G}\left(X, A, \psi ; Z_{2}\right)$, are then defined to be the homology groups of the complex $\left(C^{0}(X, A), \partial\right)$.

By using Čech [7], [5] or singular [3] methods to obtain a complex, this may be extended to all topological $G$ pairs.

Being given a compact differentiable manifold $M^{n}$ with differentiable $G$ action $\varphi$, one may triangulate $M$ "finely" so that $G$ acts simplicially. Clearly the fundamental cycle $\mu=\sum \Delta^{i}$, the sum of all $n$-simplices, is then an invariant chain, defining a fundamental class $[M, \partial M, \varphi] \in H_{n}^{G}\left(M, \partial M, \varphi ; Z_{2}\right)$. This lifts the ordinary fundamental class back to Smith theory.

One then has a natural transformation

$$
\mu: \mathfrak{N}_{*}^{G}(X, A, \psi) \rightarrow H_{*}^{G}\left(X, A, \psi ; Z_{2}\right)
$$

assigning to the equivariant bordism element $f:(M, \partial M, \varphi) \rightarrow(X, A, \psi)$ the class $f_{*}[M, \partial M, \varphi]$.

Letting $\varepsilon: \mathfrak{R}_{*}^{G} \rightarrow Z_{2}$ be the augmentation to $\mathfrak{N}_{0} \cong Z_{2}$ given by ignoring $G$ action and the positive dimensional part, one has $\mu(\alpha \cdot \beta)=\varepsilon(\alpha) \mu(\beta)$ for $\alpha \in \mathfrak{R}_{*}^{G}, \beta \in \mathfrak{R}_{*}^{G}(X, A, \psi)$ as in [9] (Note: $H_{i}^{G}\left(M^{n}, \partial M^{n}, \varphi ; Z_{2}\right)=0$ if $\left.i>n\right)$ and thus $\mu$ induces a natural transformation

$$
\tilde{\mu}: \mathfrak{N}_{*}^{G}(X, A, \psi) \otimes_{\mathfrak{N} G} Z_{2} \rightarrow H_{*}^{G}\left(X, A, \psi ; Z_{2}\right) .
$$

One has the analogue of [9, Proposition 2.1]:

LeMMA 2.1. If $G$ is a 2 group then $\tilde{\mu}$ is epic.

Proof. The result is known for $G=\{1\}$ or $G=Z_{2}$ and so one may induct on the order of $G$. Let $T=\{1, t\}$ be a central subgroup of $G$ of order 2 .

Being given a $G$ complex $(X, A, \psi)$, any element of $C^{0}(X)$ decomposes uniquely into a sum of invariant chains $\sigma_{1}+\sigma_{2}$, where $\sigma_{1}$ is a sum of simplices $\Delta$ with $t_{\#} \Delta=\Delta$ and $\sigma_{2}$ is a sum of terms $\Delta+t_{\#} \Delta$ with $t_{\#} \Delta \neq \Delta, \Delta$ a simplex. This gives a natural decomposition

and

$$
H_{*}^{G}(X, A, \psi) \cong H_{*}^{G}\left(X, F_{T} \cup A, \psi\right) \oplus H_{*}^{G}\left(F_{T}, F_{T} \cap A, \psi\right)
$$

$$
\begin{aligned}
& H_{*}^{G}\left(X, F_{T} \cup A, \psi\right) \cong H_{*}^{G / T}\left(X / T, A / T \cup F_{T}, \psi^{\prime}\right), \\
& H_{*}^{G}\left(F_{T}, F_{T} \cap A, \psi\right) \cong H_{*}^{G / T}\left(F_{T}, F_{T} \cap A, \psi^{\prime}\right)
\end{aligned}
$$

where $F_{T}$ is the fixed set of $T$ and $\psi^{\prime}$ denotes the induced action (see [9, Theorem 2.1]).

Now $\mathfrak{R}_{*}^{G / T}\left(F_{T}, F_{T} \cap A\right)$ maps onto $H_{*}^{G / T}\left(F_{T}, F_{T} \cap A\right)$ by induction, and if $f:\left(M, \partial M, \varphi^{\prime}\right) \rightarrow\left(F_{T}, F_{T} \cap A, \psi^{\prime}\right)$ is a $G / T$ bordism element representing $\alpha, f$ may 
be considered a $G$ bordism element with $T$ acting trivially, to represent $\alpha$ as an element of $H_{*}^{G}\left(X, A, \psi ; Z_{2}\right)$.

Also $\mathfrak{N}_{*}^{G / T}\left(X / T, A / T \cup F_{T}\right)$ maps onto $H_{*}^{G / T}\left(X / T, A / T \cup F_{T}\right)$ by induction, and by excision arguments as in [9, Proposition 2.1], a bordism element $f:\left(M, \partial M, \varphi^{\prime}\right)$ $\rightarrow\left(X / T, A / T \cup F_{T}\right)$ may be lifted to

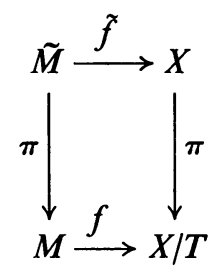

with $\tilde{f}$ being $G$ equivariant and representing the class in $H_{*}^{G}(X, A, \psi)$ corresponding to $\tilde{\mu}([f]) \in H_{*}^{G / T}\left(X / T, A / T \cup F_{T}\right)$.

If $G=\left(Z_{2}\right)^{k}$ one also has an analog of [9, Proposition 2.2].

LEMMA 2.2. If $G=\left(Z_{2}\right)^{k}$, then $\tilde{\mu}$ is an isomorphism.

Proof. This is known for $k=0,1$ and hence one may apply induction. Let $t_{1}, \ldots, t_{k}$ with $t_{i}^{2}=1, t_{i} t_{j}=t_{j} t_{i}$ be generators of $G$, with $T_{i}=\left\{1, t_{i}\right\} \subset G$.

Then for any $(X, A, \psi)$ one has an exact sequence of $\mathfrak{N}_{*}^{G}$ modules, split as $\mathfrak{R}_{*}$ modules

$$
0 \rightarrow \mathfrak{N}_{*}^{G}\left(F_{T_{1}}, A \cap F_{T_{1}}\right) \rightarrow \mathfrak{N}_{*}^{G}(X, A) \rightarrow \mathfrak{N}_{*}^{G}\left(X, F_{T_{\mathrm{i}}} \cup A\right) \rightarrow 0
$$

and hence a commutative diagram

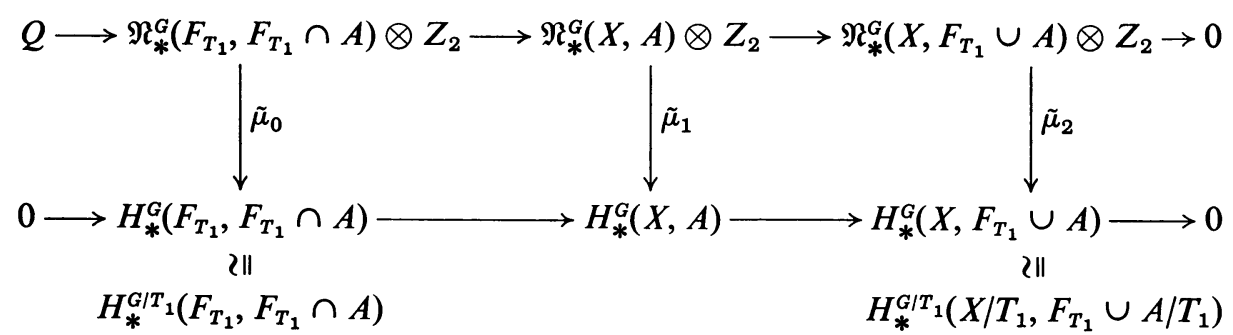

with $Q$ a "Tor"-term.

To see that $\tilde{\mu}_{2}$ is monic, one notes that $\left(X, F_{T_{1}} \cup A\right)$ is relatively free as a $T_{1}$ pair, so $\mathfrak{R}_{*}^{G}\left(X, F_{T_{1}} \cup A\right) \cong \mathfrak{N}_{*}^{G / T_{1}}\left(X / T_{1}, F_{T_{1}} \cup A / T_{1}\right)$ by assigning to a $T_{1}$ free bordism element $f: M \rightarrow X$ the induced map $\bar{f}: M / T_{1} \rightarrow X / T_{1}$. Further, this is a homomorphism of $\mathfrak{N}_{*}^{G / T_{1}}$ modules, where $\mathfrak{N}_{*}^{G / T_{1}} \rightarrow \mathfrak{N}_{*}^{G}$ by considering a $G / T_{1}$ manifold as a $G$ manifold with trivial $T_{1}$ action. One then has a commutative 
diagram

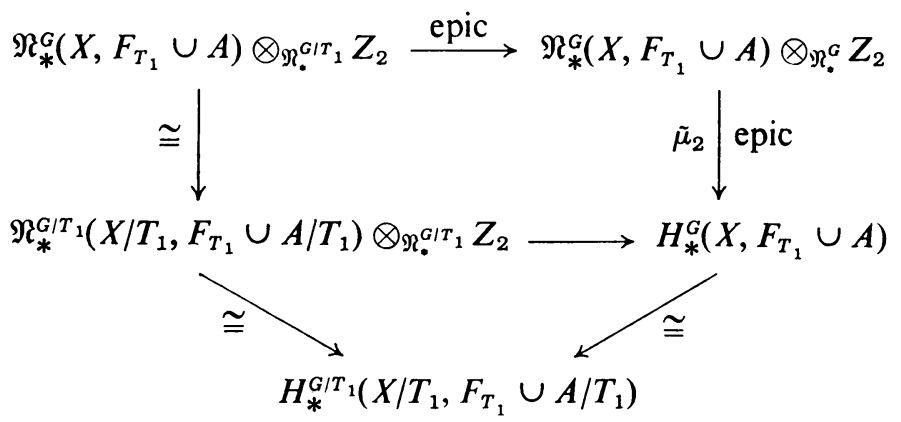

and hence $\tilde{\mu}_{2}$ is monic.

By an elementary diagram chase, $\tilde{\mu}_{1}$ will be monic provided $\tilde{\mu}_{0}$ is monic. Thus, it suffices to prove the lemma for pairs $(X, A, \psi)$ fixed by $T_{1}$. A similar analysis may then be applied to each $T_{i}$, and hence it suffices to prove the lemma for pairs $(X, A, \psi)$ fixed by each $T_{i}$, hence by $G$.

If $(X, A, \psi)$ is a trivial $G$ space, one has $\mathfrak{N}_{*}^{G}(X, A, \psi) \cong \mathfrak{N}_{*}^{G} \otimes_{\mathfrak{N}_{*}} \mathfrak{N}_{*}(X, A)$, so $\mathfrak{R}_{*}^{G}(X, A, \psi) \otimes Z_{2}$ coincides with $H_{*}\left(X, A ; Z_{2}\right)$ (the $G=\{1\}$ result) and the lemma is valid.

Now turning to the general case one has:

THEOREM 2.1. For every $G, \tilde{\mu}$ is always epic.

Proof. Let $G$ be a finite group and $(X, A, \psi)$ a $G$ pair. Let $S \subset G$ be a Sylow 2 subgroup and $\psi_{S}: S \times X \rightarrow X$ the $S$ action given by restriction to $S \times X$ of $\psi$.

Considering a $G$ invariant chain of $X$ as being only $S$ invariant defines a homomorphism

$$
\theta: H_{*}^{G}\left(X, A, \psi ; Z_{2}\right) \rightarrow H_{*}^{S}\left(X, A, \psi_{s} ; Z_{2}\right) .
$$

Being given an $S$ invariant chain $\sigma \in C(X) \otimes Z_{2}$ let $t \sigma=\sum g_{\#} \sigma$ where the sum is taken over a collection of $g$ which represent the cosets $G / S$. (Note. if $g^{\prime} \in g S$, $g_{\#}^{\prime} \sigma=g_{\#} \sigma$ since $\sigma$ is $S$ invariant.) Clearly $t \sigma$ is $G$ invariant and this induces a homomorphism

$$
t: H_{*}^{S}\left(X, A, \psi_{S} ; Z_{2}\right) \rightarrow H_{*}^{G}\left(X, A, \psi ; Z_{2}\right) .
$$

If $\sigma$ is $G$ invariant, $g_{\#} \sigma=\sigma$, so $t \sigma$ is $[G: S] \sigma$ where $[G: S]$ is the index of $S$ in $G$, and is odd, so $t \sigma=\sigma$. Thus $t \theta=1$, or $\theta$ is monic and $t$ is epic.

Now consider the extension homomorphism

$$
e_{G}^{S}: \mathfrak{R}_{*}^{S}\left(X, A, \psi_{S}\right) \rightarrow \mathfrak{R}_{*}^{G}(X, A, \psi)
$$

defined in [8, §4]. If $f:(M, \partial M, \varphi) \rightarrow\left(X, A, \psi_{S}\right)$ is an $S$ equivariant bordism element $\alpha, e_{G}^{S}(\alpha)$ is represented by $\bar{f}:(\bar{M}, \partial \bar{M}, \bar{\varphi}) \rightarrow(X, A, \psi)$ where $\bar{M}=G \times M /\left(g s^{-1}, \varphi(s, m)\right)$ $\sim(g, m), \quad \bar{\varphi}\left(g^{\prime},(g, m)\right)=\left(g^{\prime} g, m\right)$ and $\bar{f}(g, m)=\psi(g, f(m))$. If one considers 
$i: M \rightarrow \bar{M}: m \rightarrow(1, m), \bar{f} \circ i=f$ and the fundamental cycle of $\bar{M}$ is $\sum g_{\#}\left(i_{\sharp} \mu\right)$ where $\mu$ is the fundamental cycle of $M$. Thus $\bar{f}_{*}[\bar{M}, \partial \bar{M}, \bar{\varphi}]=t f_{*}[M, \partial M, \varphi]$. Thus the diagram

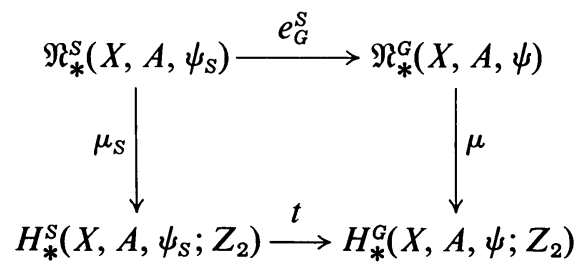

commutes, with $t$ and $\mu_{S}$ epic, so $\mu$ is epic. Hence also $\tilde{\mu}$ is epic.

Lemma 2.3. Let $G$ be a finite group with Sylow 2 subgroup $S$ and suppose the restriction $\rho_{S}^{G}: \mathfrak{N}_{*}^{G} \rightarrow \mathfrak{R}_{*}^{S}$ is epic. If $(X, A, \psi)$ is a $G$ pair with

$$
\tilde{\mu}_{S}: \mathfrak{R}_{*}^{S}\left(X, A, \psi_{S}\right) \otimes_{\mathfrak{N} S} Z_{2} \rightarrow H_{*}^{S}\left(X, A, \psi_{S} ; Z_{2}\right)
$$

monic, then

$$
\tilde{\mu}_{G}: \mathfrak{R}_{*}^{G}(X, A, \psi) \otimes_{\Re_{\odot}} Z_{2} \rightarrow H_{*}^{G}\left(X, A, \psi ; Z_{2}\right)
$$

is also monic.

Proof. Let $\rho_{S}^{G}: \mathfrak{R}_{*}^{G}(X, A, \psi) \rightarrow \mathfrak{N}_{*}^{S}\left(X, A, \psi_{S}\right)$ denote the restriction homomorphism which "ignores $G$ equivariance". It is then immediate that the diagram

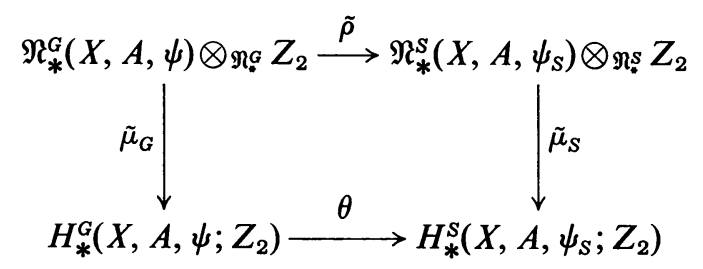

with $\tilde{\rho}$ induced by $\rho_{S}^{G}$ commutes, with $\theta$ and $\tilde{\mu}_{S}$ being monic.

Now consider the extension

$$
e_{G}^{S}: \mathfrak{R}_{*}^{S}\left(X, A, \psi_{S}\right) \rightarrow \mathfrak{N}_{*}^{G}(X, A, \psi) .
$$

By $[2,6.3] e_{G}^{S}$ is an $\mathfrak{N}_{*}^{G}$ module homomorphism; i.e. if $\alpha \in \mathfrak{N}_{*}^{S}\left(X, A, \psi_{S}\right)$ and $\beta \in \mathfrak{N}_{*}^{G}$, then $e_{G}^{S}\left(\rho_{S}^{G}(\beta) \cdot \alpha\right)=\beta \cdot e_{G}^{S}(\alpha)$. In particular, if $\beta^{\prime} \in \mathfrak{N}_{*}^{S}$, there is a $\beta \in \mathfrak{N}_{*}^{G}$ with $\rho_{S}^{G}(\beta)=\beta^{\prime}$, so $e_{G}^{S}\left(\beta^{\prime} \cdot \alpha\right)=\beta \cdot e_{G}^{S}(\alpha)$. Since $\varepsilon\left(\beta^{\prime}\right)=\varepsilon(\beta), e_{G}^{S}$ induces a homomorphism

$$
\tilde{e}: \mathfrak{N}_{*}^{S}\left(X, A, \psi_{S}\right) \otimes_{\mathfrak{M}} Z_{2} \rightarrow \mathfrak{N}_{*}^{G}(X, A, \psi) \otimes_{\mathfrak{M} q} Z_{2} .
$$

(Note. This used the fact that $\rho_{S}^{G}: \mathfrak{N}_{*}^{G} \rightarrow \mathfrak{N}_{*}^{S}$ is epic. I cannot prove that $\tilde{e}$ is meaningful without this, and in fact Theorem 2 of the Introduction would seem to imply that $\tilde{e}$ cannot always exist.)

Then $\tilde{e} \tilde{\rho}: \mathfrak{N}_{*}^{G}(X, A, \psi) \otimes_{\mathfrak{N} G} Z_{2} \rightarrow \mathfrak{R}_{*}^{G}(X, A, \psi) \otimes_{\mathfrak{N} G} Z_{2}$ is induced by $e_{G}^{S} \circ \rho_{S}^{G}$. By [8, Proposition 13.2], $e_{G}^{S} \circ \rho_{S}^{G}$ is multiplication by the class of $[G / S, \mu] \in \mathfrak{R}_{0}^{G}$, so that $\tilde{e} \tilde{\rho}$ is multiplication by $\varepsilon[G / S, \mu]=1$. 
Thus $\tilde{\rho}$ is monic, and so $\tilde{\mu}_{S} \tilde{\rho}$ is monic, which gives $\tilde{\mu}_{G}$ monic.

One then has the first half of Theorem 2 , given by

THEOREM 2.2. Let $G$ be 2-nilpotent with Sylow 2 subgroup a $Z_{2}$ vector space. Then $\tilde{\mu}$ is an isomorphism for all $G$ pairs.

Proof. Let $S$ be a Sylow 2 subgroup of $G$. Then $S=\left(Z_{2}\right)^{k}$ for some $k$, so by Lemma 2.2, $\tilde{\mu}_{S}$ is always monic. Letting $K \subset G$ be the subgroup of elements of odd order, $K$ is normal and $G / K \cong S$, giving a homomorphism $\varphi: G \rightarrow S$ with $\varphi \mid S=1$. Thus $\rho_{S}^{G}: \mathfrak{N}_{*}^{G} \rightarrow \mathfrak{P}_{*}^{S}$ is epic, for if $(M, \psi)$ is an $S$ action, $(M, \psi \circ(\varphi \times 1))$ is a $G$ action restricting to $(M, \psi)$. Thus Lemma 2.3 applies to each $G$ pair and $\tilde{\mu}_{G}$ is always monic. By Theorem 2.1, $\tilde{\mu}$ is then an isomorphism.

3. The isomorphism theorem. In order to simplify notation, temporarily say that the finite group $G$ has the isomorphism property if for all $G$ pairs $(X, A, \psi)$, the natural transformation

$$
\tilde{\mu}: \mathfrak{N}_{*}^{G}(X, A, \psi) \otimes_{\Re G} Z_{2} \rightarrow H_{*}^{G}\left(X, A, \psi ; Z_{2}\right)
$$

is an isomorphism.

Following Bredon [1], one knows that two $G$ equivariant homology theories agree for all spaces if and only if they agree for all of the coset spaces $(G / H, \mu)$, with $H$ a subgroup of $G$.

Letting $H \subset G$ be a subgroup, consider the pair $(X, A, \psi)=(G / H, \phi, \mu)$.

Clearly $C(G / H) \otimes Z_{2}$ is the $Z_{2}$ vector space with base the points of $G / H$, and these are permuted by $G$, so $C^{0}(G / H) \cong Z_{2}$ with base the sum of all the points. Thus $H_{*}^{G}\left(G / H, \mu ; Z_{2}\right) \cong Z_{2}$.

Now consider $\mathfrak{N}_{*}^{G}(G / H, \mu)$. If $f:(M, \varphi) \rightarrow(G / H, \mu)$ is a $G$ bordism element, then $M_{0}=f^{-1}(H)$, the inverse image of the coset $H$, is invariant under $H$, and hence $\left(M_{0}, \varphi \mid H \times M_{0}\right)$ is an $H$ bordism element in $\mathfrak{R}_{*}^{H}$. It is immediate that $f:(M, \varphi)$ $\rightarrow(G / H, \mu)$ is the extension to $G$ of the $H$ equivariant bordism element

$$
f \mid M_{0}:\left(M_{0}, \varphi \mid H \times M_{0}\right) \rightarrow(G / H, \mu)
$$

given by the point map. Thus, this correspondence defines an isomorphism $\mathfrak{N}_{*}^{G}(G / H, \mu) \cong \mathfrak{N}_{*}^{H}$. If $f:(M, \varphi) \rightarrow(G / H, \mu)$ and $(N, \psi) \in \mathfrak{N}_{*}^{G}$, the product is

$$
f \circ \pi_{M}:(M \times N, \varphi \times \psi) \rightarrow(G / H, \mu)
$$

so that $\left(f \circ \pi_{M}\right)^{-1}(H)=M_{0} \times N$ with action $\left(\varphi \mid H \times M_{0}\right) \times(\psi \mid H \times N)$. Thus identifying $\mathfrak{N}_{*}^{G}(G / H, \mu)$ with $\mathfrak{N}_{*}^{H}, \mathfrak{N}_{*}^{H}$ is an $\mathfrak{N}_{*}^{G}$ module by $\alpha \cdot \beta=\rho_{H}^{G}(\alpha) \cdot \beta$ for $\alpha \in \mathfrak{N}_{*}^{G}, \beta \in \mathfrak{R}_{*}^{H}$ with $\rho_{H}^{G}: \mathfrak{N}_{*}^{G} \rightarrow \mathfrak{R}_{*}^{H}$ the restriction.

Thus, one has

LEMMA 3.1. If $G$ has the isomorphism property, then for all $H \subset G$,

$$
\mathfrak{R}_{*}^{H} \otimes_{\mathfrak{M}} Z_{2} \cong Z_{2}
$$

where $\mathfrak{N}_{*}^{H}$ is an $\mathfrak{R}_{*}^{G}$ module via the restriction $\rho_{H}^{G}: \mathfrak{N}_{*}^{G} \rightarrow \mathfrak{N}_{*}^{H}$. 
LEMMA 3.2. If $H \subset G$ is a 2 group then

$$
\mathfrak{R}_{*}^{H} \otimes_{\mathfrak{N} g} Z_{2} \cong Z_{2}
$$

if and only if $\rho_{H}^{G}: \mathfrak{N}_{*}^{G} \rightarrow \mathfrak{N}_{*}^{H}$ is epic.

Proof. Clearly if $\rho_{H}^{G}$ is epic, the tensor product rule holds. Now suppose $\rho_{H}^{G}$ is not epic. Since $H$ is a 2 group $\mathfrak{N}_{0}^{H} \cong Z_{2}$ generated by a point with trivial action, which comes from a trivial $G$ action, $\left(\rho_{H}^{G}\right)_{0}$ is epic (see [8, p. 67]). Suppose

$$
\left(\rho_{H}^{G}\right)_{i}: \mathfrak{R}_{i}^{G} \rightarrow \mathfrak{N}_{i}^{H}
$$

is epic for $i<k$ and is not epic for $i=k$. Let $K=\operatorname{cokernel}\left(\rho_{H}^{G}\right)_{k}$ and let

$$
\varphi: \mathfrak{R}_{*}^{H} \rightarrow Z_{2} \oplus K
$$

be the homomorphism $\varepsilon: \mathfrak{N}_{0}^{H} \cong Z_{2}$ and quotient homomorphism $\varphi: \mathfrak{N}_{k}^{H} \rightarrow K$ and zero in all other degrees. This is clearly a vector space epimorphism.

If $\alpha \in \mathfrak{N}_{*}^{G}, \beta \in \mathfrak{N}_{*}^{H}$, consider $\varphi(\alpha \cdot \beta)=\varphi\left(\rho_{H}^{G}(\alpha) \beta\right)=x$. If $\operatorname{dim} \alpha>0, x=0$ unless $\operatorname{dim} \alpha+\operatorname{dim} \beta=k$, when $\operatorname{dim} \beta<k$. But then $\beta=\rho_{H}^{G}\left(\beta^{\prime}\right)$ for some $\beta^{\prime} \in \mathfrak{N}_{*}^{G}$, so $x=\varphi\left(\rho_{H}^{G}\left(\alpha \beta^{\prime}\right)\right)$ and represents zero in the cokernel. Thus $x=\varepsilon(\alpha) \varphi(\beta)$ if $\operatorname{dim} \alpha>0$. If $\operatorname{dim} \alpha=0, x=\varphi(\varepsilon(\alpha) \cdot \beta)=\varepsilon(\alpha) \varphi(\beta)$ for $\left(\rho_{H}^{G}\right)_{0}$ and $\varepsilon$ coincide as maps to $Z_{2}$ and $\varphi$ is $Z_{2}$ linear.

Thus, $\varphi$ induces an epimorphism $\tilde{\varphi}: \mathfrak{N}_{*}^{H} \otimes_{\mathfrak{R}} Z_{2} \rightarrow Z_{2} \oplus K$, so $\mathfrak{N}_{*}^{H} \otimes_{\Re G} Z_{2} \not Z_{2}$.

Now let $G$ have the isomorphism property, let $S \subset G$ be a Sylow 2 subgroup, and let $T \subset S$ be a central subgroup of order 2 , with $T=\{1, t\}$. By the lemmas, $\rho_{T}^{G}$ is epic, but $\rho_{T}^{G}$ is the composite

$$
\mathfrak{N}_{*}^{G} \stackrel{\rho_{S}^{G}}{\longrightarrow} \mathfrak{N}_{*}^{S} \stackrel{\rho_{T}^{S}}{\longrightarrow} \mathfrak{N}_{*}^{T}
$$

and hence $\rho_{T}^{S}$ is epic.

Now let $M^{3}$ be the manifold obtained from the 3 disc $D^{3}$ by identifying antipodal points of $S^{2}$ (i.e. RP (3)) with the $T$ action $\varphi$ given by the involution $t \cdot x=-x$ on $D^{3}$. Since $\rho_{T}^{S}$ is epic, there is an $S$ action $(N, \psi)$ cobordant to $(M, \varphi)$ as $T$ action. The fixed set of $T$ in $M$ is a point $\left(0 \in D^{3}\right)$ and RP (2) (image of $\left.S^{2}\right)$, and this is cobordant to the fixed set of $T$ in $N, F_{T}(N)$. In particular, the zero dimensional part $F_{T}(N)^{0}$ is an odd number of points. Since $T$ is normal in $S, S$ acts on $F_{T}(N)$ and hence also on $F_{T}(N)^{0}$. Since $S$ is a 2 group, each orbit of $S$ on $F_{T}(N)^{0}$ consists of $2^{r}$ points, and since $F_{T}(N)^{0}$ is odd, there must be a point orbit. Thus, there is a point $p \in F_{T}(N)^{0}$ which is fixed by $S$. Giving $N$ an $S$ invariant Riemannian metric, $S$ acts on the tangent space to $N$ at $p$ orthogonally, giving a homomorphism $\lambda: S \rightarrow O_{3}$ $(\operatorname{dim} N=3)$ and $\lambda(t)$ is multiplication by -1 in $R^{3}$. Taking the determinant 
$\operatorname{det}: O_{3} \rightarrow Z_{2}$, one has a commutative diagram

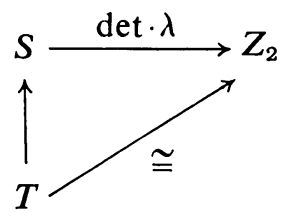

so that $T$ splits out of $S$. Since this is true for all central subgroups of order 2 in $S$, $S$ must be a $Z_{2}$ vector space. (If not, $S=A \times C, C$ the central elements of order 2, but if $A$ is nontrivial, its center is nontrivial, giving a central order 2 element of $S$ not in $C$.) Thus one has

LEMMA 3.3. If $G$ has the isomorphism property, then the Sylow 2 subgroup of $G$ is a $Z_{2}$ vector space.

In order to show that $G$ is 2-nilpotent requires a digression.

Let $G$ be a finite group and $\alpha: G \rightarrow G$ an automorphism. One lets $\alpha_{*}: \mathfrak{N}_{*}^{G} \rightarrow \mathfrak{N}_{*}^{G}$ by $\alpha_{*}(M, \varphi)=\left(M, \varphi \circ\left(\alpha^{-1} \times 1\right)\right)$, where

$$
G \times M \stackrel{\alpha^{-1} \times 1}{\longrightarrow} G \times M \stackrel{\varphi}{\longrightarrow} M
$$

defines a new $G$ action on $M$. If $\beta$ is another automorphism of $G,(\alpha \beta)^{-1} \times 1$ $=\left(\beta^{-1} \times 1\right)\left(\alpha^{-1} \times 1\right)$ so $(\alpha \beta)_{*}=\alpha_{*} \beta_{*}$, and thus one has a homomorphism

$$
*: \text { Aut }\left(G^{\prime}\right) \rightarrow \text { Aut }\left(\mathfrak{R}_{*}^{G}\right): \alpha \rightarrow \alpha_{*} \text {. }
$$

Now let $g \in G$ and $\alpha: G \rightarrow G: h \rightarrow g h g^{-1}$ the inner automorphism so that $\alpha^{-1}(h)$ $=g^{-1} h g$. Then if $(M, \varphi)$ is a $G$ action, $\alpha_{*}(M, \varphi)=(M, \psi)$ where $\psi(h, m)=\varphi\left(g^{-1} h g, m\right)$. Letting $\rho:(M, \varphi) \rightarrow(M, \psi): m \rightarrow \varphi\left(g^{-1}, m\right)$ one has an equivariant diffeomorphism, so $\alpha_{*}(M, \varphi)=(M, \varphi)$. Thus one has induced a homomorphism

$$
*: \text { Aut }(G) / \operatorname{Inn}(G) \rightarrow \operatorname{Aut}\left(\mathfrak{R}_{*}^{G}\right)
$$

where Inn $(G)$ is the normal subgroup of inner automorphisms.

Similarly, if $\alpha \in$ Aut $(G), \alpha$ acts on the set of irreducible (real) representations of $G$, IR $(G)$, by sending $\theta: G \times V \rightarrow V$ to $\theta \circ\left(\alpha^{-1} \times 1\right): G \times V \rightarrow V$. This defines a homomorphism

$$
\text { - : Aut }(G) \rightarrow \operatorname{Perm}(\operatorname{IR}(G)): \alpha \rightarrow \bar{\alpha}, \quad \text { where } \bar{\alpha}(V, \theta)=\left(V, \theta \circ\left(\alpha^{-1} \times 1\right)\right) .
$$

Notice that Inn $(G)$ acts trivially on IR $(G)$, that $\bar{\alpha}$ preserves the dimension of the representation, and $\bar{\alpha}$ sends the trivial representation $(\theta(g, v)=v$ for all $(g, v))$ to itself.

Now let $\theta: G \times V \rightarrow V$ be an irreducible real representation of $G$. Let $M$ be the manifold obtained from the disc in $V \oplus V, D(2 V)$, by identifying antipodal points 
of the sphere, with $G$ action $\varphi$ given by $\varphi\left(g,\left[v_{1}, v_{2}\right]\right)=\left[\theta\left(g, v_{1}\right), \theta\left(g, v_{2}\right)\right]$. Then the fixed set of $G$ in $(M, \varphi)$ is

$$
\begin{aligned}
F_{G}(M, \varphi) & =\{0\} & & \text { if } \operatorname{dim} V>1, \\
& =\{0\} \cup \operatorname{RP}(2 V) & & \text { if } \operatorname{dim} V=1,
\end{aligned}
$$

and $G$ acts in the normal bundle at 0 as two copies of the representation $V$. Thus letting

$$
F_{G}: \mathfrak{N}_{2 \operatorname{dim} v}^{G} \rightarrow \mathfrak{N}_{0}\left(F_{G}^{\prime}\left(B O_{2 \operatorname{dim} v}\right)\right)
$$

be the fixed point homomorphism, $F_{G}(M, \varphi)$ is given by the inclusion of a point in the component of $F_{G}^{\prime}\left(B O_{2 \operatorname{dim} V}\right)$ over which $G$ acts as $2 V$. (See [8] for the definition of $F_{G}^{\prime}\left(B O_{n}\right)$ and the fixed point homomorphism.)

It is immediate that if $(M, \varphi)$ is defined by the representation $(V, \theta)$, then $\alpha_{*}(M, \varphi)$ is defined by $\bar{\alpha}(V, \theta)$. Then if $\alpha \in \operatorname{Aut}(G)$ with $\alpha_{*}=1, F_{G}(M, \varphi)=F_{G}\left(\alpha_{*}(M, \varphi)\right)$ so that $(V, \theta)$ and $\bar{\alpha}(V, \theta)$ are equivalent representations, or $\bar{\alpha}=1$. Thus one has

Lemma 3.4. If $\alpha \in$ Aut $(G)$ and $\alpha_{*}: \mathfrak{N}_{*}^{G} \rightarrow \mathfrak{N}_{*}^{G}$ is trivial, then $\bar{\alpha}: \operatorname{IR}(G) \rightarrow \operatorname{IR}(G)$ is also trivial, or $\alpha$ acts trivially on the irreducible representations of $G$.

Now consider a finite group $G$ with the isomorphism property, and let $S$ be a Sylow 2 subgroup of $G$, so that $\rho_{S}^{G}$ is epic (Lemmas 3.1 and 3.2). If $N$ is the normalizer of $S$ in $G, S \subset N \subset G$, then $\rho_{S}^{G}=\rho_{S}^{N} \rho_{N}^{G}$, so $\rho_{S}^{N}: \mathfrak{R}_{*}^{N} \rightarrow \mathfrak{R}_{*}^{S}$ is epic.

If $n \in N, c_{n}: N \rightarrow N: g \rightarrow n g n^{-1}$ is an inner automorphism, so $\left(c_{n}\right)_{*} \in$ Aut $\left(\mathfrak{R}_{*}^{N}\right)$ is trivial. Since $S$ is normal in $N, c_{n}(S) \subset S$ and $c_{n}$ is an automorphism of $S$, so $\left(c_{n}\right)_{*} \in$ Aut $\left(\mathfrak{R}_{*}^{S}\right)$. Since $\rho_{S}^{N}$ is epic, $\left(c_{n}\right)_{*}$ is trivial in Aut $\left(\mathfrak{N}_{*}^{S}\right)$, and thus the homomorphism

$$
N \rightarrow \operatorname{Aut}\left(\mathfrak{N}_{*}^{S}\right): n \rightarrow\left(c_{n}\right)_{*}
$$

is trivial, and by Lemma 3.4

$$
N \rightarrow \operatorname{Perm}(\operatorname{IR}(S)): n \rightarrow \bar{c}_{n}
$$

is trivial.

By Lemma 3.3, $S=\left(Z_{2}\right)^{k}$, and every irreducible representation of $S$ is of the form $(R, \theta)$ where $\theta(s, v)=\bar{\theta}(s) \cdot v$, with $\bar{\theta}: S \rightarrow Z_{2}=\{+1,-1\}$. Thus $\operatorname{IR}(S)$ $=\operatorname{Hom}\left(S, Z_{2}\right)$. If $\alpha \in \operatorname{Aut}(S), \bar{\alpha}(R, \theta)=(R, \psi)$ with $\bar{\psi}(s) \cdot x=\bar{\theta}\left(\alpha^{-1} s\right) \cdot x$, so $\bar{\alpha}=1$ implies $\tilde{\theta} \circ \alpha^{-1}=\tilde{\theta}$ for all $\bar{\theta} \in \operatorname{Hom}\left(S, Z_{2}\right)$ and hence $\alpha=1$. Thus, the homomorphism

$$
N \rightarrow \operatorname{Aut}(S): n \rightarrow c_{n}
$$

is trivial, or $S$ is central in its normalizer.

One may now apply the theorem of Burnside [6, Theorem 14.3.1]: If a Sylow subgroup $P$ of $G$ is in the center of its normalizer, then $G$ has a normal subgroup $H$ which has the elements of $P$ as its coset representatives.

Thus one has 
THEOREM 3.1. If $G$ is a finite group with

$$
\tilde{\mu}: \mathfrak{N}_{*}^{G}(X, A, \psi) \otimes_{\mathfrak{N}_{*}} Z_{2} \rightarrow H_{*}^{G}\left(X, A, \psi ; Z_{2}\right)
$$

an isomorphism for all $G$ pairs $(X, A, \psi)$, then $G$ is 2-nilpotent and has Sylow 2 subgroup a $Z_{2}$ vector space.

Combining this with Theorem 2.2 gives Theorem 2 of the Introduction.

\section{REFERENCES}

1. G. E. Bredon, Equivariant cohomology theories, Lecture Notes in Math., no. 34, SpringerVerlag, Berlin and New York, 1967. MR 35 \#4914.

2. P. E. Conner and E. E. Floyd, Maps of odd period, Ann. of Math. (2) 84 (1966), 132-156. MR 34 \#3587.

3. S. Eilenberg, Homology of spaces with operators. I, Trans. Amer. Math. Soc. 61 (1947), 378-417; errata 62 (1947), 548. MR 9, 52.

4. E. E. Floyd, Orbit spaces of finite transformation groups. I, Duke Math. J. 20 (1953), 563-567. MR 15, 456.

5. - "Periodic maps via Smith theory," in A. Borel, Seminar on transformation groups, Ann. of Math. Studies no. 46, Princeton Univ. Press, Princeton, N. J., 1960. MR 22 \#7129.

6. M. Hall, Jr., The theory of groups, Macmillan, New York, 1959. MR 21 \#1996.

7. P. A. Smith, "Fixed points of periodic transformations," in S. Lefschetz, Algebraic topology, Amer. Math. Soc. Colloq. Publ., vol. 27, Amer. Math. Soc., Providence, R. I., 1942. MR 4, 84.

8. R. E. Stong, Unoriented bordism and actions of finite groups, Mem. Amer. Math. Soc. No. 103 (1970).

9. - Equivariant bordism and Smith theory, Trans. Amer. Math. Soc. 159 (1971), 417426.

University of Virginia, Charlottesville, Virginia 22901 